\title{
Cutoff values for the determination of sarcopenia and the prevalence of the condition in middle-aged women: a study from Sri Lanka
}

\author{
N Rathnayake', G Alwis ${ }^{2}$, J Lenora ${ }^{3}$, S Lekamwasam ${ }^{4}$ \\ (Index words: Cutoff value, Middle-aged women, Prevalence, Sarcopenia, Sri Lanka)
}

\begin{abstract}
Introduction There is a scarcity of studies related to sarcopenia in Sri Lanka. This partly is due to lack of cutoff values to define muscle mass, strength and physical functions.

Objective This cross-sectional study determined the cutoff values of sarcopenia measures and evaluated the prevalence of sarcopenia among middle-aged Sri Lankan women.

Methods A reference group of 117 young women (20-40 years) was used for cutoff value determination. The prevalence of sarcopenia was estimated among a group of 270 women (41-60 years). Appendicular Skeletal Muscle Mass (ASMM, kg) was measured by DXA and was adjusted for height (ASMM/height $\left.{ }^{2}\right)$ to determine Relative Skeletal Muscle mass Index $\left(\mathrm{RSMl}, \mathrm{kg} / \mathrm{m}^{2}\right)$. Hand Grip Strength (HGS, kg) and Gait Speed (GS, m/s) were also measured. Cutoff values for measures of sarcopenia were taken as 2SD below the mean value of the young reference group.
\end{abstract}

Results Mean(SD) ages of the participants; young reference group and middle-aged group were 35.5 (3.8) and 52.2 (5.8) years respectively. Cutoff values for the main three measures of sarcopenia; RSMI, HGS and GS were $5.03 \mathrm{~kg} / \mathrm{m}^{2}, 9.66 \mathrm{~kg}$ and $0.96 \mathrm{~m} / \mathrm{s}$ respectively. Prevalence of pre-sarcopenia, sarcopenia and severesarcopenia among middle-aged women were $3.0 \%, 2.2 \%$, $0.7 \%$ respectively. The corresponding values among postmenopausal women were $4.2 \%, 3.0 \%, 1.2 \%$ and among premenopausal women were $1.0 \%, 1.0 \%, 0.0 \%$.

Prevalence of sarcopenia was higher among women aged between 51-60 years and severe sarcopenia was observed only in this age group.

Conclusions This study provides cutoff values for the measures of sarcopenia for Sri Lankan women. Prevalence was higher among postmenopausal women compared to premenopausal women.

Ceylon Medical Journal 2019; 64: 9-16

DOI: http://doi.org//10.4038/cmj.v64i1.8834

\section{Background}

Sarcopenia is a syndrome characterized by progressive loss of muscle mass, muscle strength and physical performance, seen particularly in old age $[1,2]$. It characterizes an impaired state of health with a high personal health care expenditure, mobility ailments, high risk of falls and fractures, impaired ability to perform activities of daily living, debilities, loss of independence and increased risk of death [1].

The diagnosis of sarcopenia requires estimation of components of sarcopenia; muscle mass, muscle strength and physical performance [1]. Many techniques such as dual energy x ray absorptiometry (DXA), magnetic resonance imaging (MRI), computed tomography (CT) scanning, hand grip strength (HGS), peak expiratory flow rate (PEFR), gait speed (GS), timed get up and go test (TGUG) and short physical performance (SPPB) are used to measure these components [1]. Among them, DXA (for the measurement of muscle mass), HGS (measurement of muscle strength) and GS (measurement of physical performance) have been used in many studies [3-13].

Sarcopenia is categorized into three stages; 'presarcopenia', 'sarcopenia' and 'severe sarcopenia' according to the guidelines established by the European Working Group of Sarcopenia for Older People (EWGSOP) [1]. The Asian Working Group of Sarcopenia (AWGS) also follows the similar guidelines to define sarcopenia [14]. The 'pre-sarcopenia' stage is characterized by low muscle mass with preserved muscle strength and physical performance. The diagnosis of 'sarcopenia' requires the presence of either low muscle strength or physical performance in addition to the low muscle mass. In 'severe sarcopenia', all three elements are affected [1].

In women, loss of muscle mass begins in the $4^{\text {th }}$ decade and continues at a rate of $1 \%-2 \%$ per year [15] with acceleration after the age of 75 [16]. Furthermore, muscle strength decreases by $21 \%$ between 25 and 55 years in women [17]. Sarcopenia is associated with

${ }^{1}$ Department of Nursing, Faculty of Allied Health Sciences, University of Ruhuna, ${ }^{2}$ Department of Anatomy, Faculty of Medicine, University of Ruhuna, ${ }^{3}$ Department of Physiology, Faculty of Medicine, University of Ruhuna, ${ }^{4}$ Population Health Research Centre, Department of Medicine, Faculty of Medicine, University of Ruhuna, Sri Lanka.

Correspondence: NR, e-mail: <nirmala.priyanthi@gmail.com>. Received 19 December 2018 and revised version accepted 11 March 2019.

This is an open-access article distributed under the terms of the Creative Commons Attribution License, which permits unrestricted use, distribution, and reproduction in any medium, provided the original author and source are credited. 
compromised exercise capacity, activities of daily living [18], cognitive functions [19] and survival [20]. Although the Sarcopenia is a syndrome associated with aging [1], primary gonadal failure, particularly after menopause, is also a stronger determinant [21-23].

In Sri Lanka the proportion of people over 60 years has increased from 5.3\% in 1953 to $10.8 \%$ in 2003 and it is predicted to reach $25 \%$ by 2030 [24]. Expanding elderly population will increase the prevalence of age-related and degenerative diseases such as sarcopenia and osteoporosis. While the health care services of the country should be prepared to meet the health demands of increasing elderly population, there is a demand for studies related to diseases which are predominantly seen among elderly.

The EWGSOP and AWGS earlier recommended uniform HGS (20kg and $18 \mathrm{~kg}$ respectively) and GS $(0.8 \mathrm{~m} / \mathrm{s})$ cutoff values and country-specific cutoff values for RSMI in diagnosing sarcopenia [1, 14]. Usage of these uniform cutoff values may either over or under-estimate the real prevalence $[25,26]$.

Therefore, a consensus was achieved in 2010 by the EWGSOP [1] and later in 2014 by the AWGS [14] to use country specific normative reference data for all three measures, generated from healthy young adult; age between 20-40 years in order to accommodate the geographical variations of these measures. It is also recommended that cutoff values are set at two standard deviations (SD) below means of the reference population [1]. Previous studies of the Western and Asian populations have shown a wide range of cutoff values [3-13] highlighting the need of country or population specific cutoff values. Hence, development of country-specific cutoff values for sarcopenia is vital in order to facilitate future research in this area in the Sri Lankan context.

Further, it is important to know the prevalence of sarcopenia in different age categories. Though sarcopenia is a disease associated with aging, studies have shown a high prevalence of the condition in middle-aged women $[7,27,28]$ too. Therefore, this study was designed primarily to determine the cutoff values of the indices of sarcopenia for Sri Lankan women and secondly to assess the prevalence of sarcopenia in a group of middle-aged women in Sri Lanka.

\section{Materials and Methods}

\section{Study design, setting and subjects}

A community-based cross-sectional study was carried out in the community study area of the Faculty of Medicine, University of Ruhuna in Galle district, Sri Lanka, during June 2015 to January 2017. This study was designed as a part of a research project titled "Effects of menopause on bodily structure, functions and physical health".
A group of healthy community-dwelling women $(n=387)$ participated in the study. They were selected randomly using the multi-stage cluster sampling technique. The sample size for the main study was calculated based on the BMI (means and SD) between pre and postmenopausal women in a previous Chinese study [29]. The formula used for this calculation was; $\mathrm{N}=\left(\mathrm{Z}_{\alpha / 2}+\mathrm{Z}_{\beta}\right)^{2}$ * $\left(\sigma 1^{2}+\sigma 2^{2}\right) /(\mu 1-\mu 2)^{2}$, where $Z_{\alpha / 2}=95 \%$ confidence interval $=1.96, \mathrm{Z}_{\beta}=95 \%$ confidence interval $=1.96, \mathrm{Z}_{\beta}=$ $80 \%$ power $=0.84,=80 \%$ power $=0.84, \sigma 1, \sigma 2=$ standard deviations, $\mu 1-\mu 2=$ effects size/ difference between 2 means. Minimum recommended number of premenopausal and postmenopausal women in each category was 155 .

Of the sample, women aged 20-40 years were taken as the young reference group $(n=117)$ to establish the cutoff values. The adequacy of sample size to establish cutoff values were determined based on paper titled "A new approach to sample size calculation for reference interval studies" [30].

The prevalence of sarcopenia was determined among middle-aged women aged $>40$ years $(n=270)$. They were divided in to two groups based on the menopausal status; premenopausal women $(n=104)$ and postmenopausal women $(n=166)$. Further they were divided in to another two groups based on age; $41-50(n=106)$ and 51-60 $(n=164)$ years.

Menopausal status was determined based on the classification of Stages of Reproductive Aging Workshop (STRAW) [31]. Women who used thyroxin, corticosteroids, insulin, hormone replacement therapy or hormonal contraceptives were excluded from the study. Those who were pregnant or lactating, on dedicated dietary programs or supervised exercise programs (following a dietary program under the supervision of a dietician or aiming to achieve a weight or BMI target and following an exercise program under the instruction of physical instructor or aiming to achieve a weight or a BMI target) and those with chronic disease; non-communicable diseases, chronic infections, polycystic ovary syndrome or chronic major organ disease were also excluded.

\section{Measurements}

Body weight was measured to the nearest $0.1 \mathrm{~kg}$ while wearing light clothes and standing height was measured without footwear and recorded to the nearest $0.1 \mathrm{~cm}$ with calibrated Stadiometer (NAGATA, Tainan, Taiwan). All anthropometric indices were measured according to the standard protocol [32] by a single trained investigator to ensure the consistency of measurements. Muscle mass; Appendicular Skeletal Muscle Mass (ASMM, kg) was measured with a central-type DXA scanner (Hologic Discovery W, Hologic Inc, Bedford, MA, USA) adhering to the manufacturer's protocol. The procedure was carried 
out by the same technician who calibrated the device each scanning day. Analytical software provided by the DXA manufacturer was used to analyze Skeletal Muscle Mass (SMM). ASMM was calculated by the sum of SMM of all four limbs and the RSMI $\left(\mathrm{kg} / \mathrm{m}^{2}\right)$ was calculated using the following formula [5]; RSMI=ASMM in $\mathrm{kg}$ /height in meters ${ }^{2}$. Muscle strength; HGS $(\mathrm{kg})$ of the dominant side was measured [10] using the Lafayette hand held dynamometer (Lafayette Instrument Co. Ltd, Sagamore Parkway North, USA). During the test, the subjects were asked to hold the dynamometer with the dominant hand on upright position with the arm at right angles and the elbow by the side of the body [10].

Physical performance; GS (m/s) was measured by 4 meter customary paced walking; the time taken to walk the central 4 meters of a 8 meter course at usual in a selfselected pace [10]. The initial and final 2 meters were excluded from the calculation to eliminate the effects of acceleration and deceleration.

Both HGS and GS tests were done twice and the average of two measurements was used for the analyses [10].

\section{Statistical analysis}

Descriptive statistics; means (SD) or frequency (\%), were used to describe the data. Cutoff values were defined as 2SDs below mean values in the reference group [1]. The between group differences of continuous variables were compared using either independent sample $t$ test or one-way ANOVA. Prevalence figures were reported as frequency $(\%)$ and $95 \%$ confidence intervals $(\mathrm{CI})$ and they were compared with Chi square or Fisher's exact tests. Data were analysed using SPSS 20.0 and P value $<0.05$ was considered statistically significant.

\section{Ethical considerations}

All procedures of this study were performed in accordance with the ethical standards of the Helsinki declaration (1975) and its later amendments (1983). Ethical clearance for this study was obtained from Ethics Review Committee, Faculty of Medicine, University of Ruhuna, Sri Lanka [Reference number; 24.09.2014:3.2]. Written informed consent was obtained from all participants included in the study.

\section{Results}

Basic characteristics of young reference group and cutoff values of the measures of sarcopenia

Mean (SD) age of young reference group was 35.5 (3.8) years. The cutoff values for the three measures of sarcopenia; RSMI, HGS and GS were $5.03 \mathrm{~kg} / \mathrm{m}^{2}, 9.66 \mathrm{~kg}$ and $0.96 \mathrm{~m} / \mathrm{s}$, respectively (Table 1 ).

\section{Basic characteristics of middle-aged women and prevalence of sarcopenia}

Mean(SD) age of middle-aged women was 52.2(5.8) years. Postmenopausal women's Mean(SD) age at menopause was 48.3(3.9) years. Postmenopausal women were shorter when compared with premenopausal women ( $\mathrm{p}<0.001)$ (Table 2). Compared to premenopausal women, postmenopausal women had significantly lower ASMM, RSMI, HGS and GS and a higher prevalence of low RSMI, HGS and GS (Table 2).

Prevalence of pre-sarcopenia, sarcopenia and severesarcopenia in the entire study sample were $3.0 \%, 2.2 \%$, $0.7 \%$. Furthermore, the prevalence of pre-sarcopenia, sarcopenia and severe-sarcopenia among postmenopausal women were higher $(4.2 \%, 3.0 \%$ and $1.2 \%)$ compared to premenopausal women $(1.0 \%, 1.0 \%$ and $0.0 \%)(\mathrm{p}>0.05)$ (Table 2).

ASMM ( $\mathrm{p}=0.005)$ and RSMI $(\mathrm{p}=0.06)$ gradually declined with advancing age (Table 3, Figure 1A). HGS and GS also declined gradually with the advancing age (Table 3, Figure 1B and 1C), and both have improved from the age 55 years $(\mathrm{p}<0.001)$ (Figure $1 \mathrm{~B}$ and $1 \mathrm{C})$. However, the changes of HGS and GS were not significantly different ( $p>0.05)$ in the age 51-55 and 56-60 years age groups (Figure 1B and 1C).

Age related trends in the prevalence of low RSMI, HGS, GS and the three categories of sarcopenia is shown in the Table 3. Prevalence of sarcopenia was higher among women aged between 51-60 years and severe sarcopenia was observed only in this age group (Table 3).

\section{Table 1. Descriptive data of women in the young reference group and the cut off values for main three measures of sarcopenia $(n=117)$}

\begin{tabular}{lll}
\hline Parameter & Mean $(S D)$ & Cutoff value \\
\hline Age (years) & $35.5(3.8)$ & - \\
Height $(\mathrm{m})$ & $1.53(0.07)$ & - \\
Weight $(\mathrm{kg})$ & $58.14(8.90)$ & - \\
BMI $\left(\mathrm{kg} / \mathrm{m}^{2}\right)$ & $24.63(3.46)$ & $*$ \\
ASMM $(\mathrm{kg})$ & $16.01(2.36)$ & $* 5.03 \mathrm{~kg} / \mathrm{m}^{2}$ \\
RSMI $\left(\mathrm{kg} / \mathrm{m}^{2}\right)$ & $6.77(0.87)$ & $* 9.66 \mathrm{~kg}$ \\
HGS $(\mathrm{kg})$ & $21.34(5.84)$ & $* 0.96 \mathrm{~m} / \mathrm{s}$ \\
GS $(\mathrm{m} / \mathrm{s})$ & $1.26(0.15)$ & \\
\hline
\end{tabular}

*values are $2 \mathrm{SDs}$ below mean value $\mathrm{SD}=$ standard deviation, $\mathrm{BMI}=$ body mass index, ASMM= appendicular skeletal mass, RSMI=relative appendicular skeletal muscle mass index, HGS=hand grip strength, GS=gait speed 
Table 2. Basic characteristics and prevalence of low RSMI, HGS, GS and sarcopenia among middle-aged women divided according to menopausal status $(n=270)$

\begin{tabular}{|c|c|c|c|c|}
\hline $\begin{array}{c}\text { Characteristics } \\
\text { or } \\
\text { Prevalence }\end{array}$ & $\begin{array}{l}\text { Whole group } \\
\qquad(n=270)\end{array}$ & $\begin{array}{l}\text { Premenopausal } \\
\text { women } \\
(n=104)\end{array}$ & $\begin{array}{c}\text { Postmenopausal } \\
\text { women } \\
(n=166)\end{array}$ & $P$ value \\
\hline \multicolumn{5}{|c|}{ Basic characteristics; Mean (SD) } \\
\hline Age (years) & $52.4(5.6)$ & $46.9(3.2)$ & $55.8(3.8)$ & $<0.001 *$ \\
\hline Height (m) & $1.50(0.06)$ & $1.51(0.05)$ & $1.49(0.06)$ & $0.009 *$ \\
\hline Weight $(\mathrm{kg})$ & $57.44(11.31)$ & $58.00(10.30)$ & $57.09(11.91)$ & $0.52 *$ \\
\hline BMI $\left(\mathrm{kg} / \mathrm{m}^{2}\right)$ & $25.32(4.48)$ & $25.21(4.36)$ & $25.39(4.56)$ & $0.75 *$ \\
\hline ASMM (kg) & $15.34(2.82)$ & $15.99(2.57)$ & $14.87(2.96)$ & $0.003 *$ \\
\hline $\operatorname{RSMI}\left(\mathrm{kg} / \mathrm{m}^{2}\right)$ & 6.75 & $6.93(0.98)$ & $6.64(1.05)$ & $0.02 *$ \\
\hline HGS (kg) & $16.56(5.44)$ & $18.61(5.71)$ & $15.27(4.86)$ & $<0.001 *$ \\
\hline $\mathrm{GS}(\mathrm{m} / \mathrm{s})$ & $1.11(0.17)$ & $1.18(0.18)$ & $1.07(0.16)$ & $<0.001 *$ \\
\hline \multicolumn{5}{|c|}{ Prevalence; Number $(\%),(95 \% \mathrm{CI})$} \\
\hline Low RSMI & $\begin{array}{l}16(5.9) \\
(3.54-9.63)\end{array}$ & $\begin{array}{l}2(1.9) \\
(0.33-7.45)\end{array}$ & $\begin{array}{l}14 \quad(8.0 .4) \\
(4.86-14.01)\end{array}$ & $0.02 * *$ \\
\hline Low HGS & $\begin{array}{l}28(10.3) \\
(7.27-14.58)\end{array}$ & $\begin{array}{l}5(4.8) \\
(1.78-11.40)\end{array}$ & $\begin{array}{l}23(13.9) \\
(9.17-20.28)\end{array}$ & $0.01 * *$ \\
\hline Low GS & $\begin{array}{l}47(17.4) \\
(11.88-20.97)\end{array}$ & $\begin{array}{l}7(6.7) \\
(1.78-11.40)\end{array}$ & $\begin{array}{l}40 \quad(24.1) \\
(16.89-30.17)\end{array}$ & $<0.001^{* *}$ \\
\hline Pre-sarcopenia & $\begin{array}{ll}8 & (3.0) \\
(1.38-5.97)\end{array}$ & $\begin{array}{l}1(0.9) \\
(0.05-6.01)\end{array}$ & $\begin{array}{l}7(4.2) \\
(1.86-8.84)\end{array}$ & $0.11 * *$ \\
\hline Sarcopenia & $\begin{array}{l}6(2.2) \\
(0.91-5.01)\end{array}$ & $\begin{array}{l}1(0.9) \\
(0.05-6.01)\end{array}$ & $\begin{array}{l}5(3.0) \\
(1.11-7.26)\end{array}$ & $0.25 * *$ \\
\hline Severe sarcopenia & $\begin{array}{l}2(0.7) \\
(0.13-2.94)\end{array}$ & $\begin{array}{l}0(0.0) \\
(0.00-4.44)\end{array}$ & $\begin{array}{l}2(1.2) \\
(0.21-4.73)\end{array}$ & $0.37 * *$ \\
\hline
\end{tabular}

BMI=body mass index, ASMM=appendicular skeletal muscle mass, RSMI=relative skeletal muscle mass index, HGS=hand grip strength, GS=gait speed, SD=standard deviation, $\mathrm{CI}=$ confidence interval

*p value derived from independent sample t test.

** $\mathrm{p}$ value derived from Chi square or Fisher's exact tests.

Table 3. Basic characteristics and prevalence of low RSMI, HGS, GS and sarcopenia among middle-aged women divided according to age groups $(n=270)$

\begin{tabular}{cccc}
\hline Characteristics & Women age & Women age & $P$ value \\
or & between 41-50 years & between 41-50 years & \\
Prevalence & $(n=106)$ & $(n=164)$ & \\
& & & \\
\hline
\end{tabular}

Basic characteristics; Mean (SD)

$\begin{array}{ll}\text { Age (years) } & 46.4(2.6) \\ \text { Height }(\mathrm{m}) & 1.52(0.05) \\ \text { Weight }(\mathrm{kg}) & 58.49(11.25) \\ \text { BMI }\left(\mathrm{kg} / \mathrm{m}^{2}\right) & 25.32(4.64) \\ \text { ASMM }(\mathrm{kg}) & 15.97(2.73) \\ \text { RSMI }\left(\mathrm{kg} / \mathrm{m}^{2}\right) & 6.90(1.04) \\ \text { HGS }(\mathrm{kg}) & 18.94(5.47) \\ \text { GS }(\mathrm{m} / \mathrm{s}) & 1.18(0.19)\end{array}$

$\begin{array}{ll}56.2(3.2) & <0.001 * \\ 1.49(0.06) & 0.001 * \\ 56.77(11.33) & 0.22 * \\ 25.32(4.39) & 0.99 * \\ 14.94(2.80) & 0.004 * \\ 6.66(1.02) & 0.06 * \\ 15.01(4.85) & <0.001 * \\ 1.07(0.15) & <0.001 *\end{array}$

(Continued) 


\begin{tabular}{|c|c|c|c|}
\hline $\begin{array}{c}\text { Characteristics } \\
\text { or } \\
\text { Prevalence }\end{array}$ & $\begin{array}{c}\text { Women age } \\
\text { between } 41-50 \text { years } \\
(n=106)\end{array}$ & $\begin{array}{c}\text { Women age } \\
\text { between } 41-50 \text { years } \\
(n=164)\end{array}$ & $P$ value \\
\hline \multicolumn{4}{|c|}{ Prevalence; Number $(\%),(95 \% \mathrm{CI})$} \\
\hline Low RSMI & $\begin{array}{l}2(1.9) \\
(0.33-7.32)\end{array}$ & $\begin{array}{l}14(8.4) \\
(4.92-14.18)\end{array}$ & $0.02 * *$ \\
\hline Low HGS & $\begin{array}{l}4(3.8) \\
(1.21-9.94)\end{array}$ & $\begin{array}{l}24(14.6) \\
(9.78-21.19)\end{array}$ & $0.004 * *$ \\
\hline Low GS & $\begin{array}{l}10(9.4) \\
(4.86-17.06)\end{array}$ & $\begin{array}{l}37(22.6) \\
(16.57-29.87)\end{array}$ & $0.005 * *$ \\
\hline Pre-sarcopenia & $\begin{array}{l}1(0.9) \\
(0.05-5.90)\end{array}$ & $\begin{array}{l}7(4.2) \\
(1.88-8.94)\end{array}$ & $0.11 * *$ \\
\hline Sarcopenia & $\begin{array}{l}1(0.9) \\
(0.05-5.90)\end{array}$ & $\begin{array}{l}5(3.0) \\
(1.13-7.35)\end{array}$ & $0.25 * *$ \\
\hline Severe sarcopenia & $\begin{array}{l}0(0.0) \\
(0.00-4.36)\end{array}$ & $\begin{array}{l}2(1.2) \\
(0.21-4.79)\end{array}$ & $0.25 * *$ \\
\hline
\end{tabular}

BMI=body mass index, ASMM=appendicular skeletal muscle mass, RSMI=relative skeletal muscle mass index, HGS=hand grip strength, GS=gait speed, $\mathrm{SD}=$ standard deviation, $\mathrm{CI}=$ confidence interval

*p value derived from independent sample $t$ test.

$* * \mathrm{p}$ value derived from Chi square or Fisher's exact tests.

Mean RSMI $\left(\mathrm{kg} / \mathrm{m}^{2}\right)$

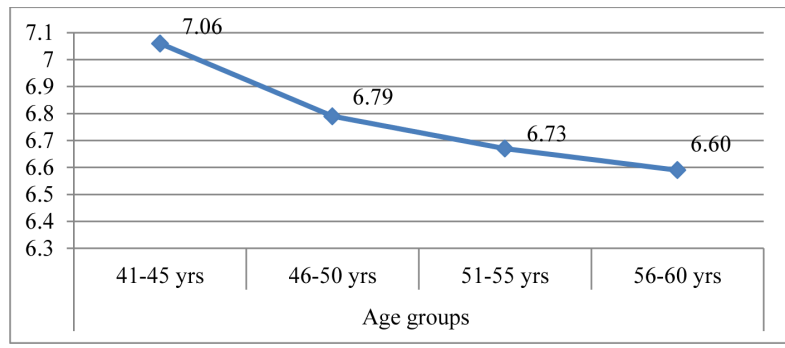

A

\section{Mean HGS (kg)}

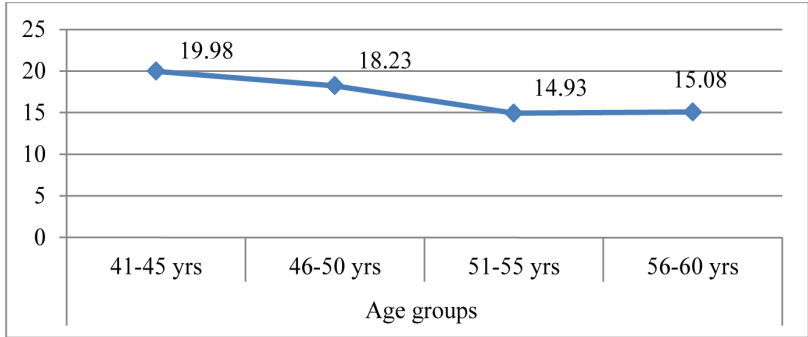

B

Mean GS (m/s)

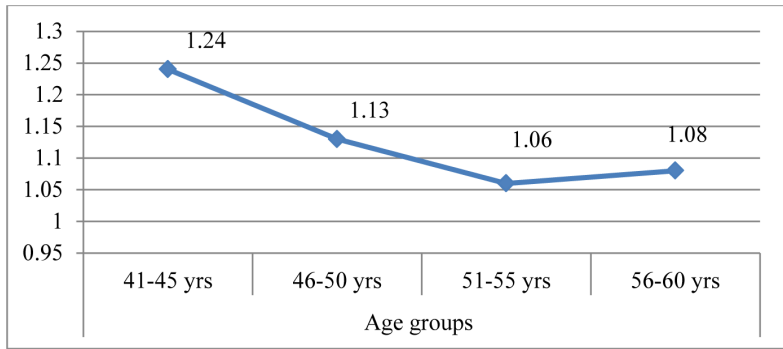

C

Figure 1. Changes of relative skeletal muscle mass index (A), hand grip strength (B) and gait speed $(C)$ with age in middle-aged women $(n=270)$. 


\section{Discussion}

This study provides the cutoff values for the measures of sarcopenia developed using a young reference group of women selected from the community. It also illustrates the prevalence of sarcopenia in middle-aged women and how it changes with age and menopausal state.

\section{Cutoff values for measures of sarcopenia}

Cutoff values for the main three measures of sarcopenia; RSMI, HGS and GS were $5.03 \mathrm{~kg} / \mathrm{m}^{2}, 9.66 \mathrm{~kg}$ and $0.96 \mathrm{~m} / \mathrm{s}$ respectively.

DXA based RSMI cutoff values ranged between $5.50-5.67 \mathrm{~kg} / \mathrm{m}^{2}$ in previous studies done among western populations [3-5]. However, many studies have used RSMI; $5.50 \mathrm{~kg} / \mathrm{m}^{2}$ developed by Baumgartner et al using US population [5] as the cutoff value to detect sarcopenia $[21,22,33]$ in the West. Studies from South-East Asia, however, have shown RSMI cutoff values of a wider range (4.82 to $6.43 \mathrm{~kg} / \mathrm{m}^{2}$ ) and many have used population specific cutoff values in their studies [6-8, 34]. Our cutoff value of $5.03 \mathrm{~kg} / \mathrm{m}^{2}$ is low compared to the Western studies but keeping with Asian values. Asians have lower RSMI cutoff values compared to non-Asians [1,14] and the difference persists even after adjusting for height [5, 35]. Further, the differences among the Asian studies might be due to the sampling characteristics; sample size, the age limit of the reference group and the method used to determine the cutoff value. Some studies have used 2SD below the mean of young reference group [6-8] similar to the current study; however, some have used lowest quartile value of study group [34] as the cutoff point for RSMI.

HGS cutoff values also vary between studies [9-13], range between $14-20 \mathrm{~kg}$ and our value of $9.66 \mathrm{~kg}$ is low compared to both Western [9, 10] and Asian [11-13] values. This difference could partly be due to the differences in defining the age limit of the reference population and the way HGS cutoff values are determined. While we have taken 20-40 years as the reference population and 2SD below mean as the cut point based on EWGSOP's guidelines, some studies have used $20^{\text {th }}$ percentile $[9,10]$ or $1^{\text {st }}$ quartile value [11-13] of the same study group as the cut point. However, genuine country or ethnic specific variations also cannot be ruled out as Tee et al [36] in Philippine who adapted a method and sample size similar to ours, found a higher HGS cutoff value of $16.1 \mathrm{~kg}$ than us.

The GS cutoff value we observed is concordant with the studies done elsewhere [10-13, 37]. Furthermore, our cutoff value of GS is close to the fixed values recommended by EWGSOP [1] and AWGS [14].

As the cutoff values of indices used in sarcopenia show a wide geographical variation, usage of country or ethnic specific cutoff values are recommended by the EWGSOP and AWGS for an accurate estimation of the prevalence of sarcopenia in a given region $[1,14]$. This, however, can be a daunting task for countries with limited resources. For such instances, the uniform cutoff values developed by the EWGSOP and AWGS [1, 14] can be used.

\section{Prevalence of sarcopenia in middle-aged women}

The prevalence of sarcopenia varies according to age and menopausal state; postmenopausal women had relatively high prevalence compared to premenopausal women and women aged 51-60 years had high prevalence compared to the other age group.

Sarcopenia prevalence was higher in postmenopausal women $(19.5 \%)$ compared to premenopausal women $(1.5 \%)$ [21] in UK. In the same study [21], sarcopenia prevalence increased with advancing age in middle-aged women. The prevalence of sarcopenia increased from $0.9 \%$ among women aged $<45$ to $3.7 \%$ among $45-50$ and $22.6 \%$ among 51-60 years. Another USA study revealed that prevalence of sarcopenia in middle-aged women; $3 \%$ in $40-49$ and $7 \%$ in 50-59 years [33]. Pongchaiyakul et al [28] reported that prevalence of sarcopenia in middle-aged women in Thailand as 3.7\% in 40-49 and $18.6 \%$ in 50-59 years and sarcopenia was only observed in women aged 50-59 years in Korea (prevalence; 0.2\%) [7].

Above studies have defined sarcopenia merely on the muscle mass (RSMI 2SD below mean) and no muscle strength or functions have been taken in to consideration. We too observed a similar change in RSMI prevalence with age and menopausal state although our values were lower compared to them [7, 21, 28]. Furthermore, the age related trend in sarcopenia prevalence has been consistent irrespective of whether the technology used was DXA [7, 21, 28] or BIA [33].

The observed variations in sarcopenia prevalence based on age and menopausal state could be due to many reasons. Sarcopenia has a complex multi-factorial etiology. Estrogen depletion, induced by exhaustion of ovarian function is associated with loss of muscle mass [22] and $10-15 \%$ extra loss of muscle strength [38] in women. Furthermore, several other mechanisms such as accumulation of free radicals, imbalanced nutrition mainly the less consumption of proteins and less physical activity may also contribute to above variations in sarcopenia prevalence [39]. The lower prevalence of sarcopenia we observed could be due to several reasons. Comparatively smaller sample size and strict sampling criteria we used may partly explain the lower prevalence rates observed. We excluded women with chronic diseases such as infections, renal and cardiac diseases, women who were on medications that can affect muscle mass or its function from our analysis, would be the other reason. However the possibility that sarcopenia is less prevalent among Sri Lankan middle-aged women cannot be excluded.

Our study has a few limitations. This analysis was based on a sample of women selected from one study area in the Southern province. This may affect the external 
validity of data and generalizability of results. We, however, selected these women in random manner to represent the population they live and applied strict inclusion and exclusion criteria to increase the internal validity of data. Also we used central-type DXA machine to measure muscle mass and the accuracy of data was ensured performing daily calibrations. Further, we used all main three criteria in defining sarcopenia and its stages. Also, we were unable to find studies of similar nature done in Sri Lanka and our data will provide a platform for future research in this increasingly important area of medicine.

\section{Conclusion}

The current study provides cutoff values for the main three measures of sarcopenia; RSMI, HGS and GS were $5.03 \mathrm{~kg} / \mathrm{m}^{2}, 9.66 \mathrm{~kg}$ and $0.96 \mathrm{~m} / \mathrm{s}$ respectively. Based on these cutoff values, low prevalence of sarcopenia was observed in the studied healthy middle-aged women. Menopause plays a significant role in the increasing of the prevalence of sarcopenia. Furthermore, we recommend future studies in this area with larger samples selected from general population of different regions of the country.

\section{Conflicts of interests}

There are no conflicts of interests.

\section{Acknowledgments}

The authors wish to express sincere gratitude to funding sources; National Research Council (Grant No 15-023), Sri Lanka and Faculty Research Committee, Faculty of Medicine, University of Ruhuna for providing funds for the study. And further, Ms. Malini Kariyawasam and Ms. Rajika Niroshini, DXA scanning unit, Teaching Hospital, Karapitiya, Sri Lanka and all the participants of the study are also acknowledged.

NB - This manuscript was derived from the corresponding author's PhD study at University of Ruhuna, Sri Lanka.

\section{References}

1. Cruz-Jentoft AJ, Baeyens JP, Bauer JM, et al. Sarcopenia: European consensus on definition and diagnosis: Report of the European Working Group on Sarcopenia in Older People. Age Ageing 2010; 39(4): 412-23.

2. Delmonico MJ, Harris TB, Visser M, et al. Longitudinal study of muscle strength, quality, and adipose tissue infiltration. The American Journal of Clinical Nutrition 2009; 90(6): 1579-85.

3. Delmonico MJ, Harris TB, Lee JS, et al. Alternative definitions of sarcopenia, lower extremity performance, and functional impairment with aging in older men and women. $J$ Am Geriatr Soc 2007; 55(5): 769-74.

4. Newman AB, Kupelian V, Visser M, et al. Sarcopenia: alternative definitions and associations with lower extremity function. J Am Geriatr Soc 2003; 51(11): 1602-9.

5. Baumgartner RN, Koehler KM, Gallagher D, et al. Epidemiology of sarcopenia among the elderly in New Mexico. Am J Epidemiol 1998; 147(8): 755-63.

6. Sanada K, Miyachi M, Tanimoto M, et al. A cross-sectional study of sarcopenia in Japanese men and women: reference values and association with cardiovascular risk factors. Eur J Appl Physiol 2010; 110(1): 57-65.

7. Kim YS, Lee Y, Chung YS, et al. Prevalence of sarcopenia and sarcopenic obesity in the Korean population based on the Fourth Korean National Health and Nutritional Examination Surveys. The Journals of Gerontology Series A, Biological Sciences and Medical Sciences 2012; 67(10): 1107-13.

8. Shimokata H, Ando F, Yuki A, et al. Age-related changes in skeletal muscle mass among community-dwelling Japanese: a 12-year longitudinal study. Geriatr Gerontol Int 2014; 14 Suppl 1: 85-92.

9. Fried LP, Tangen CM, Walston J, et al. Frailty in older adults: evidence for a phenotype. The Journals of Gerontology Series A, Biological Sciences and Medical Sciences 2001; 56(3): M146-56.

10. Lauretani F, Russo CR, Bandinelli S, et al. Age-associated changes in skeletal muscles and their effect on mobility: an operational diagnosis of sarcopenia. J Appl Physiol (1985) 2003; 95(5): 1851-60.

11. Tanimoto Y, Watanabe M, Sun W, et al. Association between sarcopenia and higher-level functional capacity in daily living in community-dwelling elderly subjects in Japan. Arch Gerontol Geriatr 2012; 55(2): e9-13.

12. Ishii $\mathrm{S}$, Tanaka $\mathrm{T}$, Shibasaki $\mathrm{K}$, et al. Development of a simple screening test for sarcopenia in older adults. Geriatr Gerontol Int 2014; 14 Suppl 1: 93-101.

13. Hong W, Cheng Q, Zhu X, et al. Prevalence of Sarcopenia and Its Relationship with Sites of Fragility Fractures in Elderly Chinese Men and Women. PLoS One 2015; 10(9): e0138102.

14. Chen LK, Liu LK, Woo J, et al. Sarcopenia in Asia: consensus report of the Asian Working Group for Sarcopenia. Journal of the American Medical Directors Association 2014; 15(2): 95-101.

15. Buford TW, Anton SD, Judge AR, et al. Models of accelerated sarcopenia: critical pieces for solving the puzzle of agerelated muscle atrophy. Ageing Res Rev 2010; 9(4): 369-83.

16. Waters DL, Baumgartner RN, Garry PJ. Sarcopenia: current perspectives. J Nutr Health Aging 2000; 4(3): 133-9.

17. Maltais ML, Desroches J, Dionne IJ. Changes in muscle mass and strength after menopause. J Musculoskelet Neuronal Interact 2009; 9(4): 186-97. 
18. Taekema DG, Gussekloo J, Maier AB, et al. Handgrip strength as a predictor of functional, psychological and social health. A prospective population-based study among the oldest old. Age Ageing 2010; 39(3): 331-7.

19. Taekema DG, Ling CH, Kurrle SE, et al. Temporal relationship between handgrip strength and cognitive performance in oldest old people. Age Ageing 2012; 41(4): 506-12.

20. Cooper R, Kuh D, Hardy R, et al. Objectively measured physical capability levels and mortality: systematic review and meta-analysis. BMJ 2010; 341: c4467.

21. Walsh MC, Hunter GR, Livingstone MB. Sarcopenia in premenopausal and postmenopausal women with osteopenia, osteoporosis and normal bone mineral density. Osteoporos Int 2006; 17(1): 61-7.

22. Iannuzzi-Sucich M, Prestwood KM, Kenny AM. Prevalence of sarcopenia and predictors of skeletal muscle mass in healthy, older men and women. The Journals of Gerontology Series A, Biological Sciences and Medical Sciences 2002; 7(12): M772-7.

23. Park HM, Ha YC, Yoo JI, et al. Prevalence of Sarcopenia Adjusted Body Mass Index in the Korean Woman Based on the Korean National Health and Nutritional Examination Surveys. Journal of Bone Metabolism 2016; 23(4): 243-47.

24. Siddhisena K. Socio-economic implications of ageing in Sri Lanka: an overview. Oxford Institute of Ageing Working Papers Oxford: Oxford Institute of Ageing 2005.

25. Bahat G, Tufan A, Tufan F, et al. Cut-off points to identify sarcopenia according to European Working Group on Sarcopenia in Older People (EWGSOP) definition. Clin Nutr 2016; 35(6): 1557-63.

26. Zeng $\mathrm{P}, \mathrm{Wu} \mathrm{S}, \mathrm{Han} \mathrm{Y}$, et al. Differences in body composition and physical functions associated with sarcopenia in Chinese elderly: Reference Values and Prevalence 60(1): $18-123$.

27. Bae EJ, Kim YH. Factors Affecting Sarcopenia in Korean Adults by Age Groups. Osong Public Health and Research Perspectives 2017; 8(3): 169-78.

28. Pongchaiyakul C, Limpawattana $\mathrm{P}$, Kotruchin $\mathrm{P}$, et al. Prevalence of sarcopenia and associated factors among Thai population. J Bone Miner Metabolism 2013; 31(3): 346-50.
29. Lin W-Y, Yang W-S, Lee L-T, et al. Insulin resistance, obesity, and metabolic syndrome among non-diabetic pre- and postmenopausal women in North Taiwan. International Journal of Obesity 2006; 30(6): 912.

30. Jennen-Steinmetz C, Wellek S. A new approach to sample size calculation for reference interval studies. Statistics in Medicine 2005; 24(20): 199-212.

31. Harlow SD, Gass M, Hall JE, et al. Executive summary of the Stages of Reproductive Aging Workshop +10 : addressing the unfinished agenda of staging reproductive aging. Menopause 2012; 19(4): 387-95.

32. Lohman TG, Roche AF, Martorell R. Anthropometric standardization reference manual: Human kinetics books, 1988.

33. Janssen I, Heymsfield SB, Ross R. Low relative skeletal muscle mass (sarcopenia) in older persons is associated with functional impairment and physical disability. $\mathrm{J} \mathrm{Am}$ Geriatr Soc 2002; 50(5): 889-96.

34. Liu LK, Lee WJ, Chen LY, et al. Sarcopenia, and its association with cardiometabolic and functional characteristics in Taiwan: results from I-Lan Longitudinal Aging Study. Geriatr Gerontol Int 2014; 14 Suppl 1: $36-45$.

35. Lau EM, Lynn HS, Woo JW, et al. Prevalence of and risk factors for sarcopenia in elderly Chinese men and women. The Journals of Gerontology Series A: Biological Sciences and Medical Sciences 2005; 60(2): 213-16.

36. Tee ML, Tee CA, Montemayor EB. Determination of normative reference for the definition of sarcopenia among Filipinos. Osteoporosis and Sarcopenia 2016; 2(3): 186-90.

37. Cesari M, Kritchevsky SB, Newman AB, et al. Added value of physical performance measures in predicting adverse health-related events: results from the Health, Aging And Body Composition Study. J Am Geriatr Soc 2009; 57(2): 251-9.

38. Samson MM, Meeuwsen I, Crowe A, et al. Relationships between physical performance measures, age, height and body weight in healthy adults. Age and Ageing 2000; 29(3): 235-42.

39. Messier V, Rabasa-Lhoret R, Barbat-Artigas S, et al. Menopause and sarcopenia: a potential role for sex hormones. Maturitas 2011; 68(4): 331-36. 